\title{
Diagnostic Accuracy Of RIPASA Scoring System In Presumptive Accurate Diagnosis Of Acute Appendicitis : A case control study
}

\author{
Mehwish Mooghal ${ }^{1}$, Wajiha Khan ${ }^{2}$, Shahid Mehmood ${ }^{1}$, Lal Bux ${ }^{1}$, Asrar Ahmad ${ }^{1}$, Kamran \\ Rahim $^{1}$, Hina Tahseen ${ }^{1}$, Amna Gul ${ }^{1}$, and Mahwish Mahboob Bhutta ${ }^{1}$ \\ ${ }^{1}$ Bahria University Medical and Dental College \\ ${ }^{2}$ Dow University of Health Sciences
}

July 27, 2021

\begin{abstract}
Introduction: The RIPASA scoring system was established specifically for Asians. Chong CF in his study consisting of 312 patients who had an emergency appendectomy concluded that optimal cut-off threshold score for negative appendectomy was 7.5. Objective: To determine the diagnostic accuracy of RIPASA scoring system in presumptive accurate diagnosis of acute appendicitis by taking histopathology as the gold standard. Materials and methods: This Cross Sectional Study was carried out at Surgery department at a tertiary care hospital, Karachi over a period of 6 months, from 15th December 2019 to 15 th June 2020. A total of 141 patients of both gender of suspected cases of acute appendicitis presenting with sign and symptoms were included in the study. Patients underwent detailed history, general and physical examination and scoring of patients according to RIPASA scoring scale and then decision of appendectomy was taken on the basis of RIPASA score. Removed appendix samples were sent for histopathology. Results: Age range in this study was from 15 to 50 years with mean age of $30.191 \pm 6.09$ years and mean RIPASA score was $5.375 \pm 1.77$. Majority of patients were males (58.9\%). RIPASA score diagnosed 28(19.9\%) and histopathology diagnosed $27(19.1 \%)$ patients with acute appendicitis. RIPASA score showed sensitivity of $85.2 \%$, specificity 95.6\%, diagnostic accuracy 94\%, PPV $82.1 \%$ and NPV was $96.4 \%$. Conclusion: We conclude that RIPASA scoring system is the scoring system of choice in diagnosis of the acute appendicitis with good sensitivity albeit a hit high and specificity albeit a bit low profile.
\end{abstract}

\section{Diagnostic Accuracy Of RIPASA Scoring System In Presumptive Accurate Diagnosis Of Acute Appendicitis : A case control study}

Abstract:

\section{Introduction:}

The Raja Isteri Pengiran Anak Saleha Appendicitis (RIPASA) scoring system was established specifically for Asian populations. Chong CF in his retrospective study consisting of 312 patients who had an emergency appendectomy at Raja Isteri Pengiran Anak Saleha Hospital, Brunei, concluded that optimal cut-off threshold score for negative appendectomy was 7.5.

\section{Objective:}

To determine the diagnostic accuracy of RIPASA scoring system in presumptive accurate diagnosis of acute appendicitis by taking histopathology as the gold standard.

\section{Materials and methods:}

This Cross Sectional Study was carried out at Surgery department at a tertiary care hospital, Karachi over a period of 6 months, from $15^{\text {th }}$ December 2019 to $15^{\text {th }}$ June 2020. A total of 141 patients of both gender 
of suspected cases of acute appendicitis presenting with sign and symptoms were included in the study. Patients underwent detailed history, general and physical examination and scoring of patients according to RIPASA scoring scale and then decision of appendectomy was taken on the basis of RIPASA score. Removed appendix samples were sent for histopathology.

\section{Results:}

Age range in this study was from 15 to 50 years with mean age of $30.191 \pm 6.09$ years and mean RIPASA score was $5.375 \pm 1.77$. Majority of patients were males (58.9\%). RIPASA score diagnosed 28(19.9\%) and histopathology diagnosed 27(19.1\%) patients with acute appendicitis. RIPASA score showed sensitivity of $85.2 \%$, specificity $95.6 \%$, diagnostic accuracy $94 \%$, PPV $82.1 \%$ and NPV was $96.4 \%$.

\section{Conclusion:}

We conclude that RIPASA scoring system is the scoring system of choice in diagnosis of the acute appendicitis with good sensitivity albeit a hit high and specificity albeit a bit low profile.

\section{Keywords:}

Acute appendicitis, Diagnosis, RIPASA score, Histopathology

What's already known about this topic?

There is scarce local and international data available for efficacy of RIPASA scoring system in diagnosing acute appendicitis, very few studies have been published to determine its effectiveness and patient application.

What does this article add?

It will add more results so that the RIPASA scoring system can be generalized for diagnosing cases of acute appendicitis presenting in emergency departments of the hospitals. This scoring system is easy to carry out and does not include costly investigations, therefore it can be implemented for use in hospitals especially in developing countries where the healthcare system is already overburdened.

\section{INTRODUCTION}

Vermiform appendix has surgical importance as it has tendency to undergo inflammation and cause acute appendicitis. Acute appendicitis is most common cause of acute abdomen in young adults and appendectomy is most frequently performed urgent abdominal procedure ${ }^{1,2}$. Epidemiologic studies have showed that approximately $50 \%(13-77 \%)$ of the population will have appendicitis in their life time ${ }^{3}$, with the peak incidence is in teens 20s, while it's rare in infancy and risk of acute appendicitis decreases after middle age. Incidence is equal among males and females before puberty and increases to $3: 2$ at age 25 , thereafter greater incidence in males decline.

No cause is yet confirmed but the factors responsible can be decreased dietary fibers and increased consumption of refined carbohydrates because incidence in developing countries is gradually increasing as they are adapting more western lifestyle ${ }^{4}$.

The diagnosis is basically clinical along with lab findings. Therefore combined scoring systems of both has devised to reduce negative appendectomy rates $(15-25 \%)^{5}$. Most widely used scoring system is Alvarado scoring system, however RIPASA scoring system is new development in recent years ${ }^{6}$.

The Raja Isteri Pengiran Anak Saleha Appendicitis (RIPASA) scoring system was established in 2008 specifically for Asian populations. Chong C F in his retrospective study consisting of 312 patients who had undergone an emergency appendectomy between October 2006 and May 2008 in Department of Surgery, Raja Isteri Pengiran Anak Saleha (RIPAS) Hospital, Brunei Darussalem, concluded that optimal cut-off threshold score for negative appendectomy was 7.5 , with a sensitivity of $88 \%$, a specificity of $67 \%$, a PPV of $93 \%$ and an NPV of $53 \%$. The negative appendectomy rate decreased significantly from $16.3 \%$ to $6.9 \%$, which was a $9.4 \%$ reduction $(\mathrm{p} \text { is } 0.0007)^{6}$. 
Another study conducted at CMH Kohat by Muhammad Qasim Butt from sept 2011 to march 2012 showed that, out of 267 patients, positive cases of acute appendicitis on histopathology were 152 and RIPASA score diagnosed 155 cases of acute appendicitis. True positive were 147, false positive 8, false negative 5, and true negative 107. Sensitivity of RIPASA score was $96.7 \%$, specificity $93.0 \%$, diagnostic accuracy was $95.1 \%$, positive predictive was $94.8 \%$ and negative predictive was $95.54 \%^{3}$.

There is scarce local data available on this topic in our region and internationally so, the aim of our study is to evaluate the diagnostic accuracy of this new scoring system for presumptive accurate diagnosis of acute appendicitis by taking histopathology as gold standard. This study might be helpful in order to eliminate negative appendectomy rates in our local population.

\section{Materials and methods}

This Cross sectional study was carried out at Surgery department of a tertiary care hospital, Karachi. The study was conducted over a duration of six months from $15^{\text {th }}$ December 2019 to $15^{\text {th }}$ June 2020. Non probability consecutive sampling technique was used. By using sample size calculator for sensitivity and specificity by Dr. Lin Naig, statistics found as follows ${ }^{3}$ :

Sensitivity $=96.7 \%$

Specificity $=93 \%$

Prevalence of acute appendicitis $=50 \%$

Margin of error for sensitivity $=4.2 \%$ and specificity $=6 \%$

The calculated sample size came out as 141. Our inclusion criteria was : (i) Both genders, Male/Female (ii) Age 15 - 50 years (iii) Suspected cases of acute appendicitis presenting with pain right iliac fossa and VAS(visual analog scale) score $=4$ or more in emergency department within 48 hours of onset of pain (score $1-3=$ no to mild pain, score $3.1-6=$ mild to moderate pain, score $6.1-10=$ moderate to severe pain). The exclusion criteria was : (i) Patient with co-morbid ( HTN , IHD , CLD , AKI , CKD , DM ) by taking detailed history (ii) Patients with history of appendectomy confirmed by examination and previous medical record (iii) Pregnant females confirmation by taking history (iv) Diagnosed cases of appendicular abscess which are confirmed by detailed examination and medical record of patients (ultrasound and CT scan). The parameters of RIPASA scoring system are : age ([?] 40 years $=1$ point; $>40$ years $=0.5$ point), gender (male $=1$ point; female $=0.5$ point), right iliac fossa $(\mathrm{RIF})$ pain $=0.5$ point, migration of pain to $\mathrm{RIF}=0.5$ point, nausea and vomiting $=1$ point, anorexia $=1$ point, duration of symptoms ([?]48 hours $=1$ point; $>48$ hours $=0.5$ point $)$, RIF tenderness $=1$ point, guarding $=2$ points, rebound tenderness $=1$ point, Rovsing's sign $=2$ points, fever $=1$ point, raised white cell count $=1$ point, negative urinalysis $=1$ point and foreign national registration identity card $=1$ point. The cut-off limit of 7.5 points was deemed as positive for acute appendicitis and was used as a diagnostic tool ${ }^{7}$.

After approval by ethical review committee, patients were selected after taking written informed consent prior to inclusion in study. Those patients presenting in Emergency with pain right iliac fossa were included. Patients underwent detailed history, general and physical examination and scoring of patients according to RIPASA scoring scale and then decision of appendectomy was taken on the basis of RIPASA score. Patients were observed by same researcher to exclude observer/interviewer bias. Patient's particulars were noted and a performa was filled and attached with admission form. Removed appendix samples were sent for histopathology (Gold Standard), for confirmation of acute appendicitis.

The data was analyzed using SPSS version 23. Mean and standard deviation was calculated for age and RIPASA score. Frequency and percentage was calculated for gender, education, findings on RIPASA and histopathology. 2x2 table was used to calculate sensitivity, specificity, PPV , NPV, diagnostic accuracy for RIPASA scoring system taking histopathology as gold standard.

Effect modifiers like age, education, socio-economic status and gender were addressed through stratification. Post stratification positive predictive value(PPV), negative predictive value(NPV), diagnostic accuracy(DA) 
, sensitivity and specificity was done.

\section{RESULTS:}

Total 141 patients included in the study. Age range in this study was from 15 to 50 years with mean age of 30.191+- 6.09 years and mean RIPASA score was 5.375+-1.77. Majority of patients were males $83(58.9 \%)$. Percentages/frequency of patients according to education status in the study showed uneducated $3(2.1 \%)$, primary education $50(35.5 \%)$, secondary education $62(44 \%)$ and those with higher educational degrees were $26(18.4 \%)$ and among socioeconomic status poor were $5(3.5 \%)$, middle class $115(81.6 \%)$ and rich were 21(14.9\%). Overall RIPASA score diagnosed 28(19.9\%) and histopathology diagnosed 27(19.1\%) patients with acute appendicitis, with a chi square score of 89.55 and p value of 0.00 and sensitivity of $85.2 \%$, specificity $95.6 \%$, diagnostic accuracy $94 \%$, PPV $82.1 \%$ and NPV was $96.4 \%$. Stratification with respect to age range (15-30 years) of RIPASA score versus histopathology $(\mathrm{n}=87)$ showed a $\mathrm{p}$ value: 0.53 , Sensitivity: $13.8 \%$, Specificity: $82.7 \%, \mathrm{DA}=48.3 \%, \mathrm{PPV}=44.4 \%, \mathrm{NPV}=48.9 \%$. Stratification with respect to age range(31-50 years) of RIPASA score versus histopathology $(\mathrm{n}=54)$ showed p value: 0.38 , Sensitivity: $29.6 \%$, Specificity: $77.7 \%, \mathrm{DA}=53.7 \%, \mathrm{PPV}=57.1 \%, \mathrm{NPV}=52.5 \%$. Stratification with respect to education status (uneducated) of RIPASA score versus histopathology $(n=3)$ showed p value 1.00, Sensitivity: $33.3 \%$, Specificity: $66.6 \%, \mathrm{DA}=50 \%, \mathrm{PPV}=50 \%, \mathrm{NPV}=50 \%$. Stratification with respect to education (primary) of RIPASA score versus histopathology $(\mathrm{n}=50)$, p value: 0.79 , Sensitivity: $20 \%$,Specificity: $82 \%, \mathrm{DA}=51 \%$, $\mathrm{PPV}=52.6 \%, \mathrm{NPV}=50.6 \%$. Stratification with respect to education (secondary) of RIPASA score versus histopathology $(\mathrm{n}=62), \mathrm{p}=0.82$, Sensitivity: $20.9 \%$, Specificity: $80.6 \%, \mathrm{DA}=50.8 \%$, PPV=52\%, NPV= $50.5 \%$. Stratification with respect to education (higher) of RIPASA score versus histopathology $(\mathrm{n}=26), \mathrm{p}=$ 0.714, Sensitivity: $15.3 \%$, Specificity: $80.7 \%, \mathrm{DA}=48.1 \%, \mathrm{PPV}=44.4 \%, \mathrm{NPV}=48.8 \%$. Stratification with respect to socioeconomic status (poor) of RIPASA score versus histopathology $(\mathrm{n}=5) \mathrm{m} \mathrm{p}=1.00$, Sensitivity: $20 \%$, Specificity: $80 \%, \mathrm{DA}=50 \%, \mathrm{PPV}=50 \%, \mathrm{NPV}=50 \%$. Stratification with respect to Socioeconomic status (middle) of RIPASA score versus histopathology $(\mathrm{n}=115)$ with $\mathrm{p}$ value $=0.737$, Sensitivity: $20 \%$, Specificity: $81.7 \%, \mathrm{DA}=50.9 \%, \mathrm{PPV}=52.2 \%, \mathrm{NPV}=50.5 \%$. Stratification with respect to Socioeconomic status (rich) of RIPASA score versus histopathology $(n=21)$, p value $=0.707$, Sensitivity: $19 \%$, Specificity: $76.1 \%, \mathrm{DA}=47.6 \%, \mathrm{PPV}=44.4 \%, \mathrm{NPV}=48.4 \%$. Stratification with respect to gender (male) of RIPASA score versus histopathology ( $\mathrm{n}=83$ ), p value: 0.58 , Sensitivity: $21.6 \%$, Specificity: $74.6 \%, \mathrm{DA}=48.2 \%$, PPV= $46.1 \%$, NPV: $48.8 \%$. Stratification with respect to gender (female) of RIPASA score versus histopathology $(\mathrm{n}=58)$ showed p value: 0.281 , Sensitivity: $17.2 \%$, Specificity: $89.6 \%, \mathrm{DA}=53.4 \%, \mathrm{PPV}=62.5 \%, \mathrm{NPV}=$ $52 \%$.

\section{Discussion:}

Acute Appendicitis is a major surgical emergency and is one of the most often admitted cases to the surgical ward. Due to its increased incidence, a ED doctor need to be at its best to correctly diagnose a case of acute appendicitis, but being the best is not enough in high capacity ED department in a major tertiary care hospital of the region. ${ }^{7}$ Timely intervention is needed to circumvent any risk of perforation, peritonitis and sepsis. To operate or not to operate is conundrum forever facing a surgeon. Appendicectomy may not be necessary in all cases of acute appendicitis as several publications show that some inflamed appendix may resolve spontaneously and others can be treated with antibiotics alone. ${ }^{8,9}$ And in case of negative appendectomy the patient undergoes unnecessary surgery. ${ }^{10}$ As a diagnostic help modalities such as Ultrasound and CT scan can be employed to help in the diagnostic process. ${ }^{9,11,13}$ Ultrasound being operator dependent have a low threshold of sensitivity and specificity. ${ }^{9,12}$ And Computer Tomography Scan has a high specificity $84 \%$ but exposes the patient to ionization radiation and incurs high cost. ${ }^{13}$ Both ultrasound and CT scan are not ideal modalities in the diagnostic process especially in emergency setting as in case of acute appendicitis and are mostly expensive or woefully unavailable in developing nations or with region with limited development. ${ }^{9}$ The Alvarado scoring system was introduced to help in diagnosing of acute appendicitis by set criterion, and it worked like a charm, reducing the number of negative appendectomies drastically. ${ }^{14}$ First introduced in 1986, Alvarado scoring system quickly gained popularity among the surgical circles and became a handy tool to have a final or a prospective say in the management plan of the patient, but this scoring system also 
had a high false positive specially in females of child bearing age, and a further modification was later on added in the form of modified Alvarado score in 1994, in which shift to the left of neutrophils was excluded ${ }^{15}$, this further improved sensitivity and decreased the false positive percentage; but the reported sensitivity and specificity of these scoring system were remained low. ${ }^{16}$ While these scoring systems came of use all around the world, over time in surgical centers of Asia, it was seen that Alvarado as well as modified Alvarado were deficient for the purpose of accurately diagnosing acute appendicitis with decreased sensitivity and specificity. ${ }^{17,18}$ In 2010, it was reported by Department of Surgery, Raja Isteri Pengiran Anak Saleha (RIPAS) Hospital, Brunei Darussalam in a retrospective analysis a new scoring system that could cater better to differentiate ethnic population with different diet. ${ }^{18}$ So was introduced RIPASA scoring system for Asian population with better sensitivity and specificity for detection of acute appendicitis was $96.2 \%$ and 85.7\% respectively when compared with RIPASA. This must be kept in mind that RIPASA scoring system has been adopted and tested now in multiple centers around Pakistan and had shown promising results ${ }^{19}$. In Kohat, Butt et al has shown that RIPASA Score had sensitivity of $96.7 \%$, specificity $93.0 \%$, diagnostic accuracy was $95.1 \%{ }^{3}$ And our study showed the same profile sensitivity and specificity, PPV, NPV, FP rate and FN rates was reported by Butt et al .

Secondarily the age groups distributions showed interesting results for a practicing surgeon to consider as high risk group being the adolescent to early twenties namely of Age 15-30, were $87(61.7 \%) .{ }^{20}$ This probably reinforces the fact that nonconforming and variable and unsafe dietary practices which are the hallmark of this age group most probably contributes to the increased incidence of acute appendicitis in the said segment of the population. ${ }^{20,21}$ Similarly it is also noted that most of the false positives arose from females in child bearing age group or married, with normal appendix ${ }^{22}$, and their complains having another primary cause (extra-appendiceal pathology) namely ruptured ovarian cyst, ovarian torsion, ectopic pregnancy ${ }^{23}$. It was further noted that the false positives, patients in whom the diagnosis for acute appendicitis was missed was in age group of 40-55 and mostly female and diabetic, adding another perspective to the issue of a multiple differentials to be excluded and females pose a difficult problem therein and always needs to be considered carefully and investigated fully in context of this latest evidence. ${ }^{24,25}$ Over all our sensitivity of RIPASA Score at a cut-off value of 7.5 was sensitivity of $85.2 \%$, specificity $95.6 \%$, diagnostic accuracy $94 \%$, PPV $82.1 \%$ and NPV was $96.4 \%$ respectively. Greatly reinforcing the confidence of this scoring system.

\section{Conclusion:}

In the lieu of the study and its results herein we conclude that RIPASA scoring system is the scoring system of choice for the doctor in ED to help in diagnosis of the acute appendicitis with good sensitivity albeit a hit high and specificity albeit a bit low profile. Furthermore, it is recommended that the age group 15-30 years be considered high risk group to be taken into account and taken on low threshold for diagnosis. Also, care must be taken to rule out all the possible differentials in women of childbearing age to keep the number of negative appendectomies in check.

\section{References:}

1. Alvarado A. A practical score for the early diagnosis of acute appendicitis. Ann Emerg Med. 1986;15(5):557-64.

2. Quartey B. Interval appendectomy in adults: a necessary evil? J Emerg Trauma Shock . 2012;5(3):213-6.

3. Butt MQ, Chatha SS, Ghumman AQ, Farooq M. RIPASA score: a new diagnostic score for diagnosis of acute appendicitis. J Coll Physicians Surg Pak. 2014;24(12):894-7.

4. Arnbjornsson E. Acute appendicitis and dietary fiber. Arch Surg. 1983;118(7):868-70.

5. Chan J, Fan KS, Mak TLA, Loh SY, Ng SWY, Adapala R. Pre-operative imaging can reduce negative appendectomy rate in acute appendicitis. Ulster Med J [Internet]. 2020 Jan 1 [cited 2021 Jul 5];89(1):25-8. Available from: https://pubmed.ncbi.nlm.nih.gov/32218624/

6. Chong CF, Thien A, Mackie AJ, Tin AS, Tripathi S, Ahmad MA, et al. Comparison of RIPASA and 
Alvarado scores for the diagnosis of acute appendicitis. Singapore Med J. 2011;52(5):340-45.

7. Mohan D, Barnato AE, Rosengart MR, Farris C, Yealy DM, Switzer GE, et al . Trauma triage in the emergency departments of nontrauma centers: an analysis of individual physician caseload on triage patterns. J Trauma Acute Care Surg 2013;74(6):1541-7.

8. Huston JM, Kao LS, Chang PK, Sanders JM, Buckman S, Adams CA, et al. Antibiotics vs. appendectomy for acute uncomplicated appendicitis in adults: Review of the evidence and future directions. Surg Infect (Larchmt) 2017;18:527-35.

9. Di Saverio S, Podda M, De Simone B, Ceresoli M, Augustin G, Gori A, et al. Diagnosis and treatment of acute appendicitis: 2020 update of the WSES Jerusalem guidelines [Internet]. Vol. 15, World Journal of Emergency Surgery. BioMed Central Ltd.; 2020 [cited 2021 Jul 5]. Available from: https://pubmed.ncbi.nlm.nih.gov/32295644/

10. Ambe PC. Negative Appendectomy. It is Really Preventable? Journal of Investigative Surgery. Taylor and Francis Ltd; Vol. 32, 2019 [cited 2021 Jul 5]. p. 474-5. Available from: https://pubmed.ncbi.nlm.nih.gov/29608342/

11. Mostbeck G, Adam EJ, Nielsen MB, Claudon M, Clevert D, Nicolau C, et al . How to diagnose acute appendicitis: ultrasound first. Insights Imaging 2016;7(2):255-63.

12. Pinto F, Pinto A, Russo A, Cuppolino F, Bracale R, Fonio P, et al . Accuracy of ultrasonography in the diagnosis of acute appendicitis in adult patients: review of the literature. Crit Ultrasound J 2013;5(Suppl 1):S2.

13. Kim DW, Suh CH, Yoon HM, Kim JR, Jung AY, Lee JS, et al. Visibility of normal appendix on CT, MRI, and sonography: A systematic review and meta-analysis. Am J Roentgenol . Vol 2018 Sep 1 [cited $2021 \mathrm{Jul}$ 5];211(3):W140-50. Available from: https://pubmed.ncbi.nlm.nih.gov/30040469/

14. Alvarado A. A practical score for the early diagnosis of acute appendicitis. Ann Emerg Med 1986;15(5):557-64.

15. Kalan M, Talbot D, Cunliffe WJ, Rich AJ. Evaluation of the modified Alvarado score in the diagnosis of acute appendicitis: a prospective study. Ann R Coll Engl 1994;76(6):418-9.

16. Diaz-Barrientos CZ, Aquino-Gonzalez A, Heredia-Montano M, Navarro-Tovar F, Pineda-Espinosa MA, Espinosa de Santillana IA. The RIPASA score for the diagnosis of acute appendicitis: A comparison with the modified Alvarado score. Rev Gastroenterol Mex . 2018 Apr 1 [cited 2021 Jul 5];83(2):112-6. Available from: https://pubmed.ncbi.nlm.nih.gov/29426650/

17. Noor S, Wahab A, Afridi G, Ullah K. Comparing Ripasa Score And Alvarado Score In An Accurate Diagnosis Of Acute Appendicitis. J Ayub Med Coll Abbottabad. 2020 Jan-Mar;32(1):38-41.

18. Khan I, Ur Rehman A. Application of Alvarado scoring system in diagnosis of acute appendicitis. J Ayub Med Coll Abbottabad 2005;17(3):41-4.

19. Khan S, Usama M, Basir Y, Muhammad S, Jawad M, Khan T, Usman A, Abbas A. Evaluation Of Modified Alvarado, Ripasa And Lintula Scoring System As Diagnostic Tools For Acute Appendicitis. J Ayub Med Coll Abbottabad. 2020 Jan-Mar;32(1):46-50.

20. Sulu B. Demographic and Epidemiologic Features of Acute Appendicitis. Append Collect Essays World Rij InTech 2012;169-78.

21. Sartelli M, Baiocchi GL, Di Saverio S, Ferrara F, Labricciosa FM, Ansaloni L, et al . Appendicitis Worldwide (POSAW). World J Emerg Surg. 2018 Apr 16;13:19. doi: 10.1186/s13017-018-0179-0.

22. Lopes Vendrami C, Xu X, McCarthy RJ, Shin JS, Goodhartz LA, Horowitz JM, Kim D, et al. Body mass index as an indicator of the likelihood of ultrasound visualization of the appendix in pregnant women 
with suspicion of appendicitis. Abdom Radiol (NY). 2020 Sep;45(9):2637-2646. PMID: 32514628.

23. Kereshi B, Lee KS, Siewert B, Mortele KJ. Clinical utility of magnetic resonance imaging in the evaluation of pregnant females with suspected acute appendicitis. Abdom Radiol (NY). 2018 Jun;43(6):1446-1455

24. Tsai SH, Hsu CW, Chen SC, Lin YY, Chu SJ. Complicated acute appendicitis in diabetic patients. Am J Surg 2008;196(1):34-9.

25. Bach L, Donovan A, Loggins W, Thompson S, Richmond B. Appendicitis in Diabetics: Predictors of Complications and Their Incidence. Am Surg. 2016 Aug;82(8):753-8.

Funding disclosure : None to declare 